\section{Gametophytic transcription for acid phosphatases in pollen of Cucurbita species hybrids}

\section{David L. Mulcahy, Richard W. Robinson, Masaaki lhara, and Richard Kesseli}

\begin{abstract}
ABSTRACr: A microslab isoelectric focusing gel is demonstrated, using single pollen grains from $F_{1}$ hybrids of Cucurbita species. When zymograms of these single pollen grains were stained to indicate the presence of acid phosphatase zones, it was seen that at least 11 out of 37 , or 29.7 percent of such zones are controlled by loci that are transcribed in the microgametophyte. These results suggest that gametophytically transcribed loci may be fairly common and also that their investigation is now technically unchallenging.
\end{abstract}

IT HAS LONG BEEN suggested that pollen, with its haploid genotype and very large population sizes, might undergo selective pressures that greatly exceed those found in other parts of the angiosperm life cycle. This possibility is a double-edged one, representing, on one hand, a vulnerability in which "a gene which greatly accelerates pollen tube growth will spread through a species even if it causes moderately disadvantageous changes in the adult plant."1 It is perhaps this that prompted some investigators $^{2}$ to suggest the number of genes actually transcribed in the pollen is probably quite small. Conversely, a gene that benefits the mature plant, if it benefits also the gametophyte, could be increased rapidly by pollen tube selection. Finally it must be considered that if genes are not transcribed in the pollen, then both deleterious and beneficial effects of pollen tube selection are precluded. Evidence that genes are transcribed in poilen comes from several sources; but many of these rely on specialized phenomena such as selfincompatibility or uniquely convenient biochemical differences such as the presence or absence of alcohol dehydrogenases or waxy versus nonwaxy starch. These phenomena have provided valuable insights into the biology of pollen but, at the same time, they raise a critical question. Does the rarity of such pollen makers indicate that much of the pollen genome is indeed inactive, or is it that we have not yet learned to detect other gametophytically transcribed loci? Mascarenhas ${ }^{3}$ has

The authors are affiliated, respectively, with the Department of Botany, University of Massachusetts, Amherst, MA 01003; the Department of Seed and Vegetable Researeh, Cornell University, Geneva, NY 14456; the Department of Biochemistry, National Institute of Genetics, Mishima, 444 Japan, and the Department of Agronomy. University of California Davis. CA 95616. This research was supported in part by NSF grant DEB 79-03685 and USDA/SEA grant 5901-0410-9-0365-0.

(C) 1981, American Genetic Association. demonstrated that there is extensive protein synthesis in pollen but he points out the possibility that some of this could be the translation of longlived messenger transcribed by sporophytic tissues.

A resolution of this issue may be provided by electrophoretic studies of pollen, using either of two approaches. The first of these, recently employed by Weeden and Gottlieb ${ }^{8}$ is to examine di- or multi-meric enzymes. If pollen from segregating sporophytes exhibits homomultimers and, unlike the sporophytes, lacks heteromultimers, then the locus in question must be transcribed post-meiotically. Products of segregation would then be capable of responding to selection.

An alternative electrophoretic test for postmeiotic expression involves the analysis of individual pollen grains. In this case, postmeiotic transcription is indicated by segregation of isozymes in pollen from heterozygous individuals. It is the latter method that is described in the present paper. Specifically, the method employs a microslab gel and isoelectric focusing.

\section{Materials and Methods}

To form a gel, a sheet of GelBond film (Bioproducts, Rockland, Maine 04841) was placed, hydrophobic side up, on a sheet of glass or other smooth surface. Two strips of plastic, each approximately $0.5 \times 4.0 \times 80.0$ $\mathrm{mm}$, were then placed on the GelBond film, parallel to each other and about $20.0 \mathrm{~mm}$ apart. On top of these plastic strips was placed a microscope slide, treated with Silane A174 (Pharmacia) to promote gel adhesion. The space between the silanized slide and the gelbond film was then filled with the polyacrylamide solution: $180 \mu \mathrm{l}$ of acrylamide and bisacrylamide stock ( $4.75 \mathrm{~g}$ acryl. $+0.25 \mathrm{~g}$ bis. in $\left.10 \mathrm{ml} \mathrm{H} \mathrm{H}_{2} \mathrm{O}\right), 30 \mu$ TEMED $(0.20 \mathrm{ml}$ TEMED in $\left.10 \mathrm{ml} \mathrm{H} \mathrm{H}_{2} \mathrm{O}\right), 180 \mu \mathrm{l}$ ammonium persulfate solution $\left(0.025 \mathrm{~g} / 10 \mathrm{ml} \mathrm{H}_{2} \mathrm{O}\right) 90 \mu \mathrm{l}$ Serva ampholytes (pH 4-9), and $0.750 \mathrm{ml} \mathrm{H}_{2} \mathrm{O}$. (This mixture is slightly modified from Ruchel ${ }^{7}$.]

Once polymerization has taken place, the gels may be used at once. This is done by lifting and turning over the gel form and either sliding or peeling the GelBond film off from the gel. Free liquid on the gel surface should be removed by touching a piece of absorbent paper to the lower edge of the upturned gel.

Single pollen grains are placed within 2-3 $\mathrm{mm}$ of one edge of the gel, moistened with an aqueous solution containing $\mathbf{0 . 0 1}$ percent of ascorbic acid, 0.01 percent of cysteine, $0.2 \mathrm{M}$ urea and 1.0 percent Serva ampholyte $(\mathrm{pH}$ $2-4)$. Within a few seconds, the grains increase diameter by approximately 30 percent and then are crushed with fine forceps. (This operation is facilitated by bringing forceps to a very sharp point with a fine grained abrasive stone.) The gel is then placed on a rubber or other nonslip surface, while strips of filter paper $\{8.0 \times 70.0 \mathrm{~mm}\}$ are moistened with $0.025 \mathrm{M}$ aspartic acid and $0.025 \mathrm{M}$ glutamic acid in water or $0.025 \mathrm{M}$ arginine and $0.025 \mathrm{M}$ lysine in $2.0 \mathrm{M}$ ethylenediamine (see Radola ${ }^{6}$ ). The aspartic-glutamic strip is placed at one edge of the gel and the arginine-lysine strip to

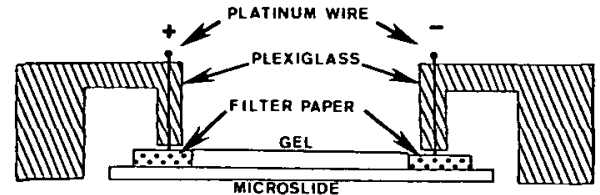

FIGURE 1 Electrophoresis apparatus. The microscope slide is $2.5 \mathrm{~cm}$ wide. See text for details.

the other. (Strips should be touching but not atop the gel.] The anode is connected to the first strip and the cathode to the other (see Figure 1). Electrophoresis is started at 100 volts and after 2-3 minutes, changed to constant power (approximately 0.5 watts). All runs are conducted at room temperature. Within 20-40 minutes, voltage increases as the conductivity of the focusing gel declines. The end of the run is signaled by a rapid increase of voltage to the maximum capability of the power supply $(500$ or $1000 \mathrm{v}$ ). No advantage is gained by using the $1000 \mathrm{v}$ setting. After focusing, the gel is placed in a standard stain for acid phosphatases (fast garnet GBC and alpha-naphthol phosphate in $0.2 \mathrm{M}$ acetate buffer, pH 5.0) for approximately 1 hour at room temperature, less at 37 C. At the end of this time (45-60 minutes) the staining solution is exhausted and thus replaced with another freshly prepared fast garnet and alpha naphthol phosphate solution. At the end of a second 45 to 60 -minute stain, gels can then be cleaned with cotton swabs, photographed and, if desired, stained for general proteins using the simplified silver method of Oakley et al. ${ }^{5}$ (It is necessary to use the pre-wash that Oakley et al. suggested for 10 percent acrylamide gels in order to remove ampholytes from the slab before staining.)

The above method is technically less demanding than the microcolumn system previously described ${ }^{4}$ and the slab configuration permits precise comparison and interpretation of samples. Also, the isoelectric focusing seems to provide a greater degree of reproducibility between separate gels than do other systems.

\section{Results and Discussion}

When single pollen grains from the $F_{1}$ hybrid, Cucurbito moschata $\times C$. polmeri, are subjected to isoelectric focusing and then stained for acid phosphatase activity, eight zones of staining are seen (Figure 2). Of these eight, six are found in all of the samples. The remaining two zones are apparently determined by a single segregating locus since each is found in approximately one of the samples. It may thus be concluded with confidence that at least two of the eight acid phosphatase zones in the pollen of this hybrid are under the control of the gametophytic genotype. As is shown, also in Figure 2, there is no evidence of segregation in any of the eight acid phosphatase zones produced from pollen of the $F_{1}$ hybrid, $C$. maxima $\times C$. moschata. Corresponding data for the $F_{1}$ between $C$. sororia $X$ C. moschata (Figure 2) are four segregating out 


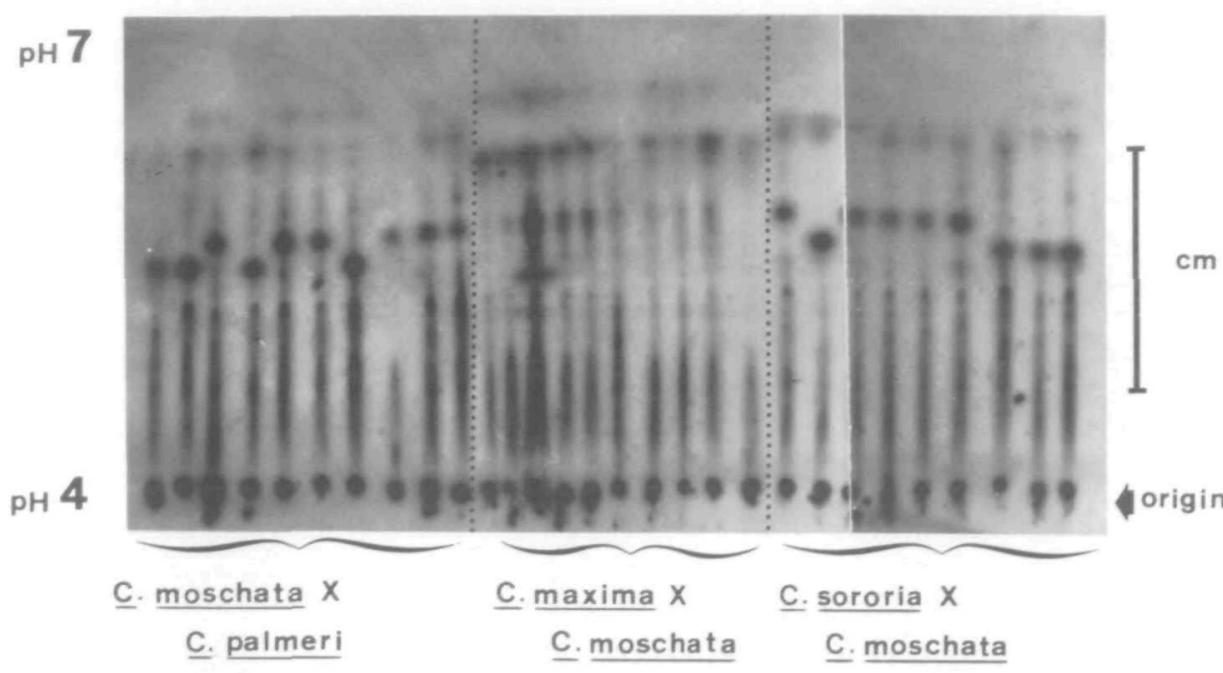

FIGURE 2 Zymograms of single pollen grains from three interspecific hybrids of Cucurbita. Both the $C$ moschata $\times$ C. palmeri and C. sororia $\times C$. moschata exhibit segregation in the pH range of 5.5 . The latter hybrid is segregating also at $\mathrm{pH}$ 6.5. The pollen from $C$. maxima $\times$ C. moschata hybrid shows no segregation for acid phosphatases in this gel.

of a total of seven. For C. texana $\times$ C. palmata (not illustrated), three out of seven, and for $C$. pepo $\times C$. texana (not illustrated), two out of seven. In all, 11 out of the 37 , i.e., 29.7 percent of the acid phosphatase zones observed in these gels were definitely the products of gametophytic transcription. Furthermore, it must be considered that this method indicates gametophytic transcription for heterozygous possible to determine the origin of the 26 other the products of zones. Some or all may be they may be products of gametophytically transcribed loci that are not segregating in this material. These results thus provide a con- servative estimate of gametophytic transcription. Nonetheless, they suggest that such transcription may be quite extensive. They also demonstrate that it is now possible to undertake a detailed investigation of gametophytic genetic activity.

\section{References}

1. Haldane, J. B. S. The Causes of Evolution. Longmans, Green, and Co., London. 1932.

2. Heslop-HARRISON, J. Summary and perspectives in higher plants as monitors of environmental mutagens. Environ. Health Perspect. 27:197-206. 1978.

3. Mascarenhas, J. P. The biochemistry of angiosperm pollen development. Bot. Rev. 41: 259-314. 1975

4. MULCAHY, D. L.., G. B. MulCAHY, and R. W. ROBINSON. Evidence for postmeiotic genetic activity in pollen of Cucurbita species. I. Hered. 70:365-368. 1979.

5. OAKLEY, B. R., D. R. KIRSCH, and N. R. MORRIS A simplified ultrasensitive silver stain for detecting proteins in polyacrylamide gels. Anal. Biochem. 105:361-363. 1980.

6. RADOLA, B. J. Ultrathin-layer isoelectric focusing in polyacrylamide gels. Electrophoresis 1:43-56. 1980.

7. RUCHEL, R. Two-dimensional micro-separation technique for proteins and peptides, combining isoelectric focusing and gradient gel electrophoresis. I. Chromatog. 132:451-468. 1977.

8. WEEDEN, N. F and L. D. GOTTLIEB, Distinguishing allozymes and isozymes of phosphoglucoisomerases by electrophoretic comparisons of pollen and somatic tissues. Biochem. Genet. 17:287-296. 1979.
The Journal of Heredity 72: 354-355. 1981.

\section{A compensating trisomic in pearl millet}

\section{R. S. Saini and J. L. Minocha}

ABSTRACT: A compensating trisomic was isolated from the progeny of a multiple interchange trisomic in pearl millet, Pennisetum typhoides. It showed the character istic morphological features of trisomy and formed a chain-of-seven chromosomes with the absence of a ring-of-four-or-six chromosomes.

ANEUPLOIDS, particularly trisomics, have been extensively used in genetic analyses and mapping of chromosomes of diploid plant species $^{1,3}$. In pearl millet, Pennisetum ty-

The authors are, respectively, scientist (S-1), Indian Council of Agricultural Research, New Delhi: and senior geneticist, Punjab Agricultural University, Ludhiana, India. This study is based on research conducted by the senior author in partial fulfillment of the requirements for the Ph.D. degree. Please address reprint requests to $\mathrm{Dr}$. Minocha. (C) 1981, American Genetic Association. phoides $[2 n=14\}$, an important cereal of semiarid regions, a complete series of primary trisomics, a number of interchange stocks and tertiary trisomics are available $e^{2,6,7}$. Compensating trisomics in which one missing chromosome is compensated for by two modified chromosomes, have not been reported in pearl millet. However, such trisomics have been successfully used for locating genes on particular arms of chromosomes in the tomato 4 . The present communication is the first report on the origin, isolation, and cytological behavior of a compensating trisomic in pearl millet.

A compensating trisomic was isolated from the progeny of a multiple interchange trisomic. The origin of multiple interchange trisomics was described by Minocha and $B r a r^{5}$. The expected types of aneuploids arising from the progeny of multiple interchange trisomics are shown in Figure 2, where ' $n$ ' designates the haploid chromosome complement with standard or interchanged chromosomes. Chromosome composition of the extra dose in the gametes and the zygotes is also given. For simplicity, the male gametes are shown with only the standard chromosome complement. One of the five combinations (square 3) involving the union of a normal male gamete ( $n$ chromosome number) with a female gamete $(n+1$ chromosome number) possessing two modified chromosomes in place of normal chromosome (1S.1L) results in the compensating trisomic. This was confirmed cytologically by the presence of a chain configuration of seven chromosomes $\left(1_{\mathrm{VII}}+4_{\mathrm{II}}\right)$ and the absence of a ring-of-fouror-six chromosomes (Figure $1 A$ ). The meiotic analysis of the compensating trisomic showed a chain of seven chromosomes in 14.4 percent of the cells. The other chromosome associa-

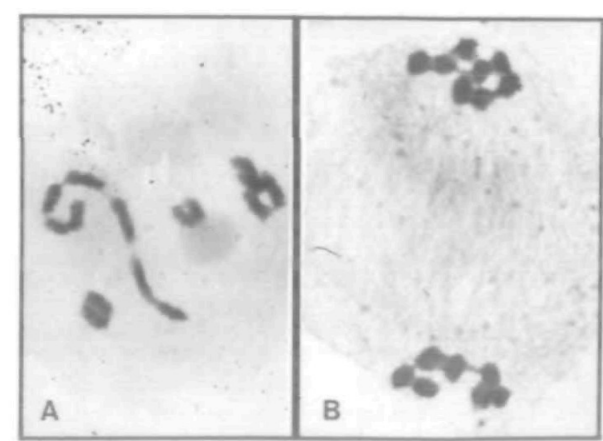

FIGURE 1 A-diakinesis in a compensating trisomic showing a chain of 7 chromosomes. B-anaphase I with 8-7 chromosome distribution in the compensating trisomic. 